\title{
Mitteilungen der Deutschen Gesellschaft für
}

\section{Transfusionsmedizin und Immunhämatologie}

Mitteilungen der Deutschen Gesellschaft für Transfusionsmedizin und Immunhämatologie HCV-lnfektionen durch Anti-D-Prophylaxe auch in der Bundesrepublik? Ein Aufruf zum Sammeln der notwendigen Daten

Liebe Kolleginnen und Kollegen, in den siebziger Jahren wurden in der DDR fast 2500 Frauen durch eine kontaminierte Anti-DImmunglobulinprophylaxe mit Hepatitis-C-Virus (HCV) infiziert [1]. In Irland wurde kürzlich entdeckt, daß, ebenfalls in den siebziger Jahren, Anti-D-Immunglobulin zu mehr als $700 \mathrm{HCV}$ Infektionen geführt hat [2]. Aufgrund unserer Untersuchungen sehen wir die Möglichkeit, daß HCV durch Anti-D-Immunglobulin-Prophylaxe auch in der Bundesrepublik übertragen wurde. Dieser Verdacht gründet sich auf eine Untersuchung an 208544 norddeutschen Blutspendern. Dort fand sich ein höherer Anteil RIBA-positiver Frauen, eine bei den Frauen im Gegensatz zu den Männern mit dem Alter deutlich steigende AntiHCVPrävalenz (RIBA) und

ein im Vergleich zu den Männern signifikant höherer Anteil rhesus-negativer, RIBA-positiver Frauen (Tab. 1).

Tab. 1. Geschlecht und Rhesusfaktor aller bis zum 31.8.1994 im DRK-Blutspendedienst in Springe anti-HCV-RIBA-positiven Blutspender (angegeben ist die Anzahl der HCV-positiven Blutspender) [aus 3]

\section{Rhesusfaktor}

D-positiv

D-negativ

Summe

Geschlecht Männlich Weiblich

171135

$24(12,3 \%) 43(24,2 \%)$

195

178 
Es ist nun sehr wichtig, die Zahlenbasis für diesen Anfangsverdacht zu erweitern. Dazu bräuchten wir aus möglichst alien Blutspendediensten der Bundesrepublik von alien dort identifizierten, RIBA(oder einem entsprechenden Test)-positiven Blutspendern eine Liste mit:

Bitte vergessen Sie nicht Ihren Namen, die Anschrift des Blutspendedienstes und eine Telefonund Faxnummer, unter der Sie zu erreichen sind, und senden Sie diese Liste an:

Dr. Gregor Caspari

Institut für Medizinische Virologie

Frankfurter Straße 107

35392 Gießen

Tel. (0641)702-2876

Fax (0641) 702-73 90

Selbstverständlich werden alle Einsender von uns über das Ergebnis informiert werden. Herzlichen Dank für Ihre Mitarbeit.

Geschäfísstelle:

Prof. Dr. Reinhold Eckstein

Abteilung für

Transfusionsmedizin

und Hämostaseologie

Uníversitätsklinikum

Erlangen/Nürnberg

Krankenhausstraße 12

D-91054Erlangen

Tel. 09131/856346

$09131 / 856987$

265

II

Mitteilungen der Literatur

Deutschen Gesellschaft

$1 / 8$

$\tau \lambda \cdot \quad \cdot \cdot 1$ Dittmann S, Roggendorf M, Dürkop J, Wiese M, Lorbeer B, Deinhardt F: Longterm persistence of hepatitis

Transtusionsmedizin ,...., ‘, ,’ 111 ÜQin,,,17

C virus in a single source outbreak. J Hepatol 1991;13:323-327. UПd Immunhämat 1 10gl̈e 2

Power JP, Lawlor E, Davidson F, Yap PL, Kenny-Walsh E, Whelton MJ, Walsh TJ: Hepatitis C viraemia in recipients of Irish intravenous anti-D immunoglobulin. Lancet 1994;344:1166-1167. 3 Caspari G, Gerlich WH, Beyer J, Schmitt H: Age, sex and transaminase dependency of specific and nonspecific results from enzyme immunoassays for antibodies to hepatitis $\mathrm{C}$ virus and follow-up of blood donors. Infusionsther Transfusionsmed 1995;22:208-219.

Geschäftsstelle:

Prof. Dr. Reinhold Eckstein Abteilung für Transfusionsmedizin und Hämostaseologie Universitätsklinikum Erlangen/Nürnberg Krankenhausstraße 12 D-91054Erlangen Tel. $09131 / 85634609131 / 856987$

I

Mitteilung der DGTI-Sektion 2 «Plasmatische Blutbestandteile > der Sitzung vom 23. März 1995 
Obmann: R. Kotitschke (Biotest Pharma GmbH, Dreieich)

Mitteilungen der

Deutschen Gesellschaft für Transfusionsmedizin und Immunhämatologie

Wissenschaftliche Vorträge

G. Caspari (Institut für medizinische Virologie, Gießen): «Aktuelle Maßnahmen zur Verbesserung der Virussicherheit von Blutprodukten»

H. Dichtelmüller (Biotest Pharma GmbH, Dreieich): Anforderungen des Paul-Ehrlich-Institutes an Arzneimittel aus Blut (Bundesanzeiger vom Mai und August 1994)

Arbeiten der Sektion

Neue Ringversuche

1. Infektionssicherheit von Blut- und Plasmaprodukten

Bis zur nächsten Sitzung der Sektion können Vorschläge an den Obmann der Sektion und/oder an den Obmann dieser Arbeitsgruppe (G. Caspari) über konkrete Vorhaben zu diesem Thema eingereicht werden.

Bisheriger Vorschlag: GPT-Ringversuch.

G. Caspari ist bereits jetzt besonders an Plasmen von HBV- und/oder HCV-positiven, noch nicht AK-positiven Spendern interessiert. Interessenten können sich direkt an ihn wenden (Tel. 06 41/7 02-28 76; Fax -73 90).

2. Ringversuch «FFP»

Der geplante Ringversuch «Gefrorenes Frischplasma» wurde anhand der Vorhabensbeschreibung diskutiert, nachdem festgestellt worden war, daß eine Finanzierung dieses Ringversuches nur mit Mitteln der Teilnehmer verwirklicht werden kann.

Vorhabensbeschreibung

1. Bezeichnung und Gesamtziel des Projektes «Gefrorenes Frischplasma»

-Teilziel 1: Herstellung von drei verschiedenen gefrorenen Frischplasmapools für mindestens 15

Teilnehmer eines Ringversuches zu dessen Charakterisierung. Herstellung eines gefriergetrockneten Referenzplasmas aus einem Frischplasmapool.

- Teilziel 2: Charakterisierung der Proteine der gefrorenen Frischplasmapools mit

biochemi-

schen und immunologischen Methoden sowie ihrer funktionellen Aktivitäten.

- Teilziel 3: Prüfung der Lagerstabilität der drei gefrorenen Frischplasmapools über einen

Zeitraum von 3 Jahren bei vier verschiedenen Lagertemperaturen: 1) -20 (C 2)-25 $\left.5^{\circ} \mathrm{C} 3\right)-30^{\circ} \mathrm{C} 4$ )$40^{\circ} \mathrm{C}$.

2. Arbeítsplan

Antikörpertiter:

Proteine:

Gerinnungsfaktoren:

Akti vierungsprodukte:

Inhitiboren (funktionell):

Komplementfaktoren:

Prüfzeitpunkte:

Prüfparameter für das zu etablierende Referenzpoolplasma sind:

A, B, 0, Rh, Anti-HBV, HBsAg, anti-HCV, SGPT, anti-HBs

Gesamtprotein, 2dimensionale Immunelektrophorese, IgG

Fibrinogen, FV, FVIII, FIX, FXП, Quick-Wert

FPA (Fibrinopeptid A), TAT (Thrombin-AT III-Komplex) 
AT III, Cl-Esterase-Inhibitor, $\alpha 2-$ Makroglobulin $\mathrm{C} 3, \mathrm{C} 4$

0, 3, 6,12,18,24, 36 Monate.

Geschäftsstelle:

Prof. Dr. Reinhold Eckstein

Abteilung für

Transfusionsmedizin

und Hämostaseologie

Universitätsklinikum

Erlangen/Nürnberg

Krankenhausstraße 12

D-91054 Erlangen

Tel. 09131/856346

$09131 / 856987$

267 